\title{
Engaging community pharmacists in the primary prevention of cardiovascular disease: protocol for the Pharmacist Assessment of Adherence, Risk and Treatment in Cardiovascular Disease (PAART CVD) pilot study
}

Kevin P Mc Namara ${ }^{1,2^{*}}$, Johnson George ${ }^{1}$, Sharleen L O'Reilly ${ }^{3}$, Shane L Jackson ${ }^{4}$, Gregory M Peterson ${ }^{4}$, Helen Howarth ${ }^{4}$, Michael J Bailey ${ }^{5}$, Gregory Duncan ${ }^{1,6}$, Peta Trinder ${ }^{1}$, Elizabeth Morabito ${ }^{1}$, Jill Finch", Stephen Bunker ${ }^{2}$, Edward Janus ${ }^{2,7}$, Jon Emery ${ }^{8}$, James A Dunbar ${ }^{2}$

\begin{abstract}
Background: Cardiovascular disease (CVD) is the leading cause of death globally. Community pharmacist intervention studies have demonstrated clinical effectiveness for improving several leading individual CVD risk factors. Primary prevention strategies increasingly emphasise the need for consideration of overall cardiovascular risk and concurrent management of multiple risk factors. It is therefore important to demonstrate the feasibility of multiple risk factor management by community pharmacists to ensure continued currency of their role.

Methods/Design: This study will be a longitudinal pre- and post-test pilot study with a single cohort of up to 100 patients in ten pharmacies. Patients aged 50-74 years with no history of heart disease or diabetes, and taking antihypertensive or lipid-lowering medicines, will be approached for participation. Assessment of cardiovascular risk, medicines use and health behaviours will be undertaken by a research assistant at baseline and following the intervention ( 6 months). Validated interview scales will be used where available. Baseline data will be used by accredited medicines management pharmacists to generate a report for the treating community pharmacist. This report will highlight individual patients' overall CVD risk and individual risk factors, as well as identifying modifiable health behaviours for risk improvement and suggesting treatment and behavioural goals. The treating community pharmacist will use this information to finalise and implement a treatment plan in conjunction with the patient and their doctor. Community pharmacists will facilitate patient improvements in lifestyle, medicines adherence, and medicines management over the course of five counselling sessions with monthly intervals. The primary outcome will be the change to average overall cardiovascular risk, assessed using the Framingham risk equation.
\end{abstract}

Discussion: This study will assess the feasibility of implementing holistic primary CVD prevention programs into community pharmacy, one of the most accessible health services in most developed countries.

Trial registration: Australia and New Zealand Clinical Trial Registry Number: ACTRN12609000677202

\footnotetext{
* Correspondence: kevin.p.mcnamara@monash.edu

'Centre for Medicine Use and Safety, Department of Pharmacy Practice, Faculty of Pharmacy and Pharmaceutical Sciences, Monash University, 381 Royal Parade, Parkville, VIC 3052, Australia

Full list of author information is available at the end of the article
} 


\section{Background}

Cardiovascular disease (CVD) is the leading cause of death globally, accounting for an estimated 17.1 million deaths per annum - 29\% of all deaths [1]. The relative contribution of CVD to the burden of disease remains high in countries with low-, middle-, and high incomes [1]. Despite significant gaps in research, there is sufficient evidence (particularly from developed nations such as the United States and United Kingdom) to suggest that the primary prevention of CVD represents a cost effective approach to reducing this burden [2].

There is a cluster of highly prevalent CVD risk factors in many developing and most developed countries including Australia which can be controlled or avoided: tobacco smoking, hypertension, overweight and obesity, physical inactivity, dyslipidaemia (high cholesterol), poor nutrition and diabetes $[1,3]$. Significant evidence-treatment gaps remain in the management of each of these, culminating in substantially elevated incidences of CVD in most countries. Community pharmacists are highly accessible health professionals in most communities and have demonstrated an effective role in the management of several of these risk factors [4-7]. Guidelines on primary CVD prevention now highlight the importance of overall CVD risk assessment rather than traditional individual risk factor assessment, resulting in a much greater emphasis on multiple risk factor management [8-13].

The expertise of community pharmacists in medicines management including adherence screening and management, and a growing role in health promotion has resulted in several studies demonstrating effective pharmacist involvement with multiple CVD risk factor management [14-16]. However, we are not aware of any multiple risk factor study specifically examining structured, pharmacist-led disease state management programs for primary CVD prevention in a non-diabetic population. The Pharmacist Assessment of Adherence, Risk and Treatment in Cardiovascular Disease (PAART $C V D$ ) was developed as a pilot project to address this evidence gap.

This paper describes the research protocol developed for the PAART CVD project, a community-pharmacy based CVD primary prevention pilot project in a nondiabetic, adult population. We also identify the theoretical framework, local issues affecting implementation decisions, and gaps in current knowledge experienced to facilitate future adaptation.

\section{Goals of research}

1. To assess the feasibility of implementing a primary CVD prevention program in a community pharmacy setting
2. To measure changes to cardiovascular health for patients receiving the PAART CVD intervention.

\section{Methods and Design}

\section{Study design and setting}

This is a longitudinal pre- and post-test study to be undertaken with a single cohort of patients. Ten pharmacies from two States (Victoria and Tasmania) will be recruited - five each from rural and metropolitan locations. Each pharmacy will be asked to approach a convenience sample of patients meeting the inclusion criteria, with a goal of recruiting 10 participants per pharmacy. Local general practices and general practice organisations will be informed about the project.

\section{Outcomes examined}

The primary outcome for the intervention will be the average change to estimated 5-year risk of primary onset of CVD between baseline and six months calculated using the New Zealand CVD risk scores [11], a modified version of the Framingham-based risk equation [17]. Secondary health outcomes include change to blood pressure, lipid profile, random blood glucose, waist, body mass index (BMI), and depression score.

\section{Pharmacist training}

Over two consecutive days, pharmacists from recruited pharmacies will attend training focussed on the following key topics:

- health promotion and behavioural change

- overall CVD risk and CVD risk assessment

- medicines adherence

- medicines management for cardiovascular risk factors

- lifestyle modification (diet, physical activity, weight management, alcohol consumption)

- antiplatelet use for primary CVD prevention

- smoking cessation

- managing patients who screen positively for diabetes, alcohol abuse or depression; and

- General Practitioner (GP) and other health professional engagement.

All training, including training materials, will be consistent with relevant national guidelines and will be tailored to suit a generalist community pharmacist audience. Emphasis will be placed on the need for pharmacists to act within their professional competencies and take appropriate action if situations arise outside their competencies (e.g. patient screens positive for depression). Written guidelines will be provided to 
identify patients with complex or urgent clinical needs that warrant referral to a GP or another health professional (e.g. dietitian). Pharmacists will also be advised on options for referral (e.g. how to access specialist dietary or smoking cessation advice) when they might deem it appropriate.

\section{Subject eligibility criteria}

Inclusion criteria

To be eligible for participation in the study, patients need to be

- aged 50-74 years

- currently taking one or more medicines for cholesterol and/or hypertension, and

- free from established cardiovascular disease, diabetes or a cardiovascular event.

Individuals will be excluded from the study in the following instances:

- patients with a complex debilitating coexisting medical condition

- target organ damage

- any cognitive impairment

- reliance on a carer or living in a residential aged care facility

- recent hospital inpatients who were medical admissions and discharged less than four weeks prior to recruitment

- non-English speaking

- living more than $40 \mathrm{~km}$ from a participating pharmacy

- patients who have received a Home Medicines Review (HMR) in the past 12 months

- deemed inappropriate for the intervention by the patient's GP; or

- patients of GPs who express a desire not to have any involvement with this project.

\section{Patient recruitment}

Pharmacists will initially be asked to conduct a simple pre-screening of potential participants based on the inclusion criteria outlined above. The information required (CVD history, use of CVD medicines, and age) is quick and easy to obtain from dispensing records and patient interview. Potential participants expressing interest will be provided with written patient information and an Expression Of Interest (EOI) form to be completed and returned to researchers.

Upon receipt of EOIs or direct patient-initiated contact via a free telephone number, a formal assessment of all eligibility requirements will be undertaken by researchers and a provisional baseline assessment date agreed. If eligible, consent must be obtained to contact their GP in writing and to provide the GP with an opportunity to comment on the appropriateness of participation. Written consent for participation and sharing of health information with relevant health professionals is to be provided by patients prior to baseline assessment.

\section{Patient data collection}

Baseline and final ( 6 month) patient information will be collected by trained research assistants (RAs). This process will take 60-90 minutes on both occasions and can take place in the patient's home (preferred), or in a private area at the pharmacy or their workplace. Assessment involves anthropometric and Point Of Care (POC) biomedical testing, and an administered questionnaire using validated scales where possible. Lipid profiles and fasting blood glucose levels will be obtained using Cholestech $\mathrm{LDX}^{\bullet}$ Analyzers $[18,19]$, in accordance with instructions provided by the manufacturer. This device uses finger-prick testing and provides results within 10 minutes. Quality control samples will be used to validate the machine prior to measurements. Patients will be advised that the most accurate results for cholesterol and blood glucose levels would be obtained by fasting 12-16 hours prior to the initial assessment interview and testing, and to take their medications as normal on the day of clinical assessment. Fasting time will be recorded to aid clinical interpretation.

When the patient is rested, blood pressure (BP) will be measured using an Omron $1 \mathrm{~A}^{\circ} \mathrm{B}^{\circ}$ automated $\mathrm{BP}$ monitor in accordance with WHO MONICA Monitoring of Trends and Determinants of Cardiovascular Diseases and the European Health Risk Monitoring protocols $[20,21]$ amended for use with an automated monitor. The average of two measurements taken 2-3 minutes apart will be used, but if systolic BP measurements differ by $10 \mathrm{mmHg}$ or greater, or diastolic BP differ by 6 $\mathrm{mmHg}$ or greater, a third reading will be taken. In this instance the average of the two closest systolic and two closest diastolic BP measurements will be used to define the BP. The RA will advise patients to seek immediate medical attention if systolic BP is $180 \mathrm{mmHg}$ or greater, or if diastolic BP is $110 \mathrm{mmHg}$ or greater. Weight, height, and waist and hip circumferences will be measured in accordance with the EHRM protocol [21].

The remainder of the assessment will consist of a researcher-administered questionnaire (all interviewing researchers have pharmacy qualifications). This questionnaire, which includes several validated survey instruments, covers a range of health indicators:

- demographic and social information, including some questions based on the WHO MONICA 
(MONItoring of CArdiovascular events) protocol [21], as adapted for the Greater Green Triangle Chronic Disease Risk Factor Studies [22]

- brief patient medical history and family history of CVD events

- details of current medicines

- details of recent testing for CVD risk factors prior to baseline

- adherence to current medications advice using the Tool for Adherence Behaviour Screening (TABS) [23] and the Morisky scale [24]

- smoking, physical activity and weight management status - including some screening questions from the 'Lifescripts' program [25] and questions from surveys used by the Greater Green Triangle Chronic Disease Risk Factor Studies [22]

- nutrition and adherence to national dietary guidelines, based on Dietary Guidelines for Australian Adults (2003) [26] and using a number of questions from the National Nutrition Survey 1995 [27], and the Cancer Council of Victoria Food Frequency Questionnaire [28]

- screening for excess alcohol consumption using the AUDIT C tool [29]

- screening for depression using the Center for Epidemiological Study of Depression Scale (CES-D) [30] - quality of life measure using the SF-12v2 Health Survey $^{\text {mix }}$ (Ware et al, 2007) [31].

Pharmacists will be asked to supply medicines dispensing data for the six months' period prior to baseline data collection and the six months prior to follow up data collection. To account for medicines collected elsewhere, patients will be asked during their interviews to recall any quantities of medicines obtained elsewhere for the specified period.

\section{Intervention part one: accredited pharmacist review}

Using relevant national clinical guidelines as the benchmark for management, pharmacists accredited to undertake collaborative, structured medicines management reviews in community settings (known as 'Home Medicines Reviews') will produce a written report for each patient highlighting the estimated 5-year CVD risk, suboptimal CVD risk factors, and medication adherence and other medicines use issues (see additional file 1 for template). The report will also suggest patient treatment goals and opportunities for beneficial lifestyle changes and improved medicines management to achieve goals. This report will be provided to the patient's community pharmacist with summaries for the patient and their general practitioner.
Intervention part two: community pharmacist facilitating patient change

Community pharmacists will be trained to deliver their interventions in accordance with the Health Action Process Approach (HAPA) [32] to behaviour change over five counselling sessions conducted at monthly intervals. The emphasis in counselling progresses from change motivation initially (via improved self-efficacy, belief in the need for change and belief that change will generate positive outcomes), through to change maintenance and relapse prevention strategies. Written, achievable goals will be encouraged.

The first session with the patient will prioritise basic health education regarding individual CVD risk and the benefits of potential treatments. It also establishes acceptable goals for the treatment process through patient consultation, and how these might be achieved. Finally, the pharmacist will discuss with the patient any specific medication changes identified in the baseline report that are recommended to improve adherence to CVD guidelines. Community pharmacists will not be trained or asked to make interventions related specifically to diabetes or mental health issues, but will be alerted to any suboptimal assessment results in these areas and asked to discuss with the patient the potential need for GP input. Such issues will also be identified in the baseline assessment summary provided to the GP.

If a patient's overall 5-year CVD risk score is 5\% or less (considered very low risk) they will be advised to discuss with their pharmacist whether they are likely to benefit from continuing with the intervention. The decision to continue will be left to the pharmacist and the patient. Pharmacists will be expected to assess and document patient motivation to undertake various medication and lifestyle changes. Following discussion with each patient, the pharmacist will then forward the clinical summary to the patient's GP with any additional comments considered relevant.

Subsequent sessions will involve: ensuring necessary changes to medicines have been made; monitoring of medicines adherence especially for new medicines; linking patients with local health and other services that provide relevant patient support; initiating lifestyle change and supporting maintenance and relapse prevention. Throughout these sessions, patient progress towards goals will be continually reassessed, as will be the goals themselves. GP input to patient treatment plans will also be invited.

\section{Primary outcomes}

The primary outcome for the PAART CVD intervention is the average change to overall estimated 5-year risk of 
CVD onset, calculated using the New Zealand adaptation of the Framingham-based CVD risk score $[11,17]$.

\section{Secondary outcomes}

Other outcomes examined include:

- Changes to individual modifiable CVD risk factors (blood pressure, lipid profile, Body Mass Index, waist circumference, smoking status, depression)

- changes in medicines adherence assessed using Morisky [24] and TABS scales [23]; and

- changes in key health behaviours (physical activity, diet, alcohol intake, weight management).

Additional outcomes to be assessed include stakeholder satisfaction (GP, pharmacist, patient) and process audit based on community pharmacist documentation of counselling sessions.

\section{Sample size}

With 80 subjects this study would have a $90 \%$ power to show an absolute reduction in risk of $1 \%$ based on a standard deviation in the change of risk of $2.7 \%$ with a two sided p-value of 0.05 . To account for potential subject drop out and loss to follow-up of $20 \%$ we will aim to recruit 100 subjects.

\section{Statistical analysis}

Univariate comparisons between groups will be conducted using chi-square test for equal proportion (or Fisher's exact tests where numbers are small) and reported as numbers and percentages. Continuous normally distributed variables will be compared using Student's t-tests and reported as means (standard error) whilst non-parametric data will be compared using Wilcoxon rank-sum tests and reported as medians (interquartile range). Pairwise differences between pre and post values will be calculated using paired t-tests for normally distributed data and Wilcoxon sign rank tests for non-normally distributed data. To account for the large number of comparisons being made, we will employ a reduced 2-sided p-value of 0.01 to indicate clear statistical significance.

If any parameter from POC finger prick testing is found to be outside the measurable range for the machine, the value of the closest measurable limit will be given to that patient.

Health behaviours will largely be measured using existing scales associated with the survey instruments incorporated into baseline and follow-up assessment. For the dietary assessment, no validated tools are available for use in an Australian population so a study-specific tool has been devised. This tool is a 13-item questionnaire addressing dietary quality in this study
Table 1 Scoring mechanism used to assess overall quality of dietary intake

\begin{tabular}{ll}
\hline $\begin{array}{l}\text { Diet score using } \\
\text { nutrition tool }\end{array}$ & Interpretation \\
\hline $104-130$ & $\begin{array}{l}\text { High level of compliance with CVD dietary } \\
\text { guidelines (80\% or more) }\end{array}$ \\
\hline $78-103$ & $\begin{array}{l}\text { Medium level of compliance with CVD dietary } \\
\text { guidelines (60-79\%) }\end{array}$ \\
\hline 77 or less & Low level of compliance with CVD dietary \\
& guidelines (59\% or less) \\
\hline
\end{tabular}

population and using previously validated questions from the National Nutrition Survey and Cancer Council of Victoria Food Frequency Questionnaire relating to the nutrients of concern for primary prevention of CVD $[27,28]$. Responses for each item will be scored between zero (least healthy response option) and 10 (healthiest option) - consistent with the developing literature in this area of nutrition research [33]. This scoring allows for an overall diet score to be generated ranging from 0 to 130 .

The general quality of dietary intake will be interpreted using the guidance provided in table 1 .

This score allows a good estimate of the level of adherence to key diet-related behaviours. Constituent elements of the 13-item questionnaire also allowed development of sub-scales for these areas of significant influence on cardiovascular health:

- fruit and vegetable intake (soluble fibre and antioxidants)

- saturated fat and total fat intake

- omega-3 fatty acid intake

- fibre intake (insoluble fibre); and

- salt intake.

The sub-scales enable community pharmacists to deliver more tailored dietary advice to participants.

The study protocol was approved by Monash University Human Research Ethics Committee (HREC), University of Tasmania HREC, Flinders University Social and Behavioural Research Ethics Committee and the Australia and New Zealand Clinical Trials Registry (Trial Number ACTRN12609000677202).

\section{Discussion}

The protocol and methods outlined above provide an intervention framework for managing multiple CVD risk factors in community pharmacy or other primary care settings. There is considerable incentive to demonstrate feasibility and effectiveness of such a service in community pharmacy, given the caution with which expanded roles for pharmacists in vascular care have been greeted by some [34]. While there is evidence of pharmacists' 
ability to conduct cardiovascular risk assessments and deliver basic post-screening counselling with some clinical benefits [35-37], evidence is lacking to demonstrate the full potential of their comprehensive, ongoing management of multiple CVD risk factors. This has led to concerns about fragmentation of care and lack of communication of results with doctors [34].

The HAPA model underpinning the approach to behavioural change was chosen because of its success in lifestyle-oriented diabetes prevention programs addressing similar risk factors for non-diabetic populations, including an implementation study based in Australian primary care $[38,39]$. Collection of process audit data is important to see how well this approach is adhered to by pharmacists. The emphasis on use of assessment scales for medicines adherence and various health behaviours is designed to promote ease of interpretation of clinical assessments by community pharmacists who are generalists in terms of their practice.

Several aspects of the protocol are context-specific and their adoption by other researchers or health service program managers may require consideration. Key issues include:

- The restriction of eligibility to patients aged 50-74 years is in keeping with national guidelines advocating that screening of overall risk outside the 45-74 years age bracket is not economically viable [9]

- The use of accredited pharmacists has been adopted to integrate with existing pharmacy skills networks and remuneration systems, and to alleviate time pressure for participating community pharmacists; there may be alternative workforce options to produce clinical reports in different settings.

- Patients with established diabetes and CVD will be excluded because there are existing evidence-based models of care already in practice or at implementation stage; and

- Community pharmacy represents a key setting and pharmacists are one of the most accessible healthcare providers in Australia, with expected competencies in both medicines use and health promotion.

\section{Limitations of study protocol}

If the pilot testing indicates the feasibility of effective community pharmacy interventions, it will be important to validate this study with larger numbers and a control group to comprehensively determine effectiveness, costeffectiveness and generalisability. Similarly, the six month timeframe limits the ability to test the sustainability of patient behaviour changes following the intervention, and self-report - while often the only option for some health behaviours may be unreliable.
Validated dietary quality tools for cardiovascular health are not available for the Australian population, so we rely on a pilot with only face and content validity developed by one of the authors (SOR). Current Australian guidelines for lipid disorders claim a lack of evidence regarding appropriate cholesterol targets for primary CVD prevention, thus restricting guidance for practitioners. We relied on expert consensus developed from examination of eligibility criteria for subsidised lipid lowering medicines in Australia, previous guidelines, and values used for pathology lab reporting.

\section{Conclusions}

The proposed intervention integrates guideline evidence and validated assessment tools where possible, but several key knowledge gaps remain to preventing an entirely evidence-informed approach. Protocols adopted for other primary CVD prevention studies have not been widely disseminated; hence this paper provides a starting point for future research and implementation programs in a primary care setting.

\section{Additional material}

Additional file 1: Clinical reporting form for baseline patient information. This document is the template used by consultant pharmacists to report baseline findings to pharmacists and doctors. Forms are first provided to the community pharmacist to allow them to add any comments before sending to the doctor. Examples of comments about different aspects of patient health behaviours and treatment are also provided.

\section{Acknowledgements}

The authors thank Professor Graham Giles of the Cancer Epidemiology Centre of The Cancer Council Victoria, for permission to use the Dietary Questionnaire for Epidemiological Studies (Version 2), Melbourne: The Cancer Council Victoria, 1996. This project, and KM's position at institution (2) is funded by the Australian Government Department of Health and Ageing as part of the Fourth Community Pharmacy Agreement through the Fourth Community Pharmacy Agreement Grants Program managed by the Pharmacy Guild of Australia. We would also like to acknowledge the contributions of the community and accredited consultant pharmacists in this study.

\section{Author details}

${ }^{1}$ Centre for Medicine Use and Safety, Department of Pharmacy Practice, Faculty of Pharmacy and Pharmaceutical Sciences, Monash University, 381 Royal Parade, Parkville, VIC 3052, Australia. ${ }^{2}$ Greater Green Triangle University Department of Rural Health, Flinders and Deakin Universities, Deakin University, Princes Hwy, Warrnambool, VIC 3280, Australia. ${ }^{3}$ Centre for Physical Activity and Nutrition Research, Faculty of Health Medicine Nursing \& Behavioural Sciences, Deakin University, 221 Burwood Highway, Burwood, Victoria 3125, Australia. ${ }^{4}$ Unit for Medication Outcomes Research and Education, School of Pharmacy, University of Tasmania, Churchill Avenue, Sandy Bay, TAS 7005, Australia. 'Department of Epidemiology and Preventive Medicine, Monash University, Alfred Hospital, Commercial Road, VIC 3004, Australia. ${ }^{6}$ Peter James Centre, Eastern Health Clinical School, Faculty of Medicine, Nursing and Health Sciences, Monash University, Mahoney's Road, Burwood East, Victoria 3151 Australia. 'Department of Medicine, University of Melbourne, Western Hospital, Gordon Street, Footscray VIC 3011. ${ }^{8}$ School of Primary, Aboriginal and Rural Health Care. The University of Western 
Australia (M501), 35 Stirling Highway, Crawley, Western Australia 6009 Australia.

\section{Authors' contributions}

KM is lead investigator and wrote the first draft with input from SOR, JD and JG. All authors provided input with subsequent drafts and read and approved the final manuscript.

\section{Competing interests}

The authors declare that they have no competing interests.

Received: 17 May 2010 Accepted: 7 September 2010

Published: 7 September 2010

\section{References}

1. Lopez AD, Begg S, Bos E: Global Burden of Disease and Risk Factors. In Demographic and Epidemiological Characteristics of Major Regions, 1990-2001. Edited by: Lopez AD, Mathers CD, Ezzati M, Jamison DT, Murray CJL. Oxford: Oxford University Press/World Bank; 2006: [http://files.dcp2.org/pdf/GBD/ GBD.pdf], Downloaded 28 April 2010.

2. Schwappach D, Boluarte T, Suhrcke M: The economics of primary prevention of cardiovascular disease - a systematic review of economic evaluations. Cost Eff Resour Alloc 2007, 5(1):5.

3. Australian Institute of Health and Welfare: Australia's Health 2008. Canberra 2008

4. Machado M, Bajcar J, Guzzo GC, Einarson TR: Sensitivity of Patient Outcomes to Pharmacist Interventions. Part II: Systematic Review and Meta-Analysis in Hypertension Management. Ann Pharmacother 2007, 41(11):1770-81.

5. Tsuyuki R, Johnson J, Teo K, Ackman M, Biggs R, Cave A, Chang WC, Dzavik V, Farris KB, Galvin D, Semchuk W, Simpson SH, Taylor JG: Study of Cardiovascular Risk Intervention by Pharmacists (SCRIP): a randomized trial design of the effect of a community pharmacist intervention program on serum cholesterol risk. Ann Pharmacother 1999, 33(9):910-19.

6. Blenkinsopp A, Anderson C, Armstrong M: Systematic review of the effectiveness of community pharmacy-based interventions to reduce risk behaviours and risk factors for coronary heart disease. J Public Health 2003, 25(2):144-53.

7. Sinclair HK, Bond CM, Stead LF: Community pharmacy personnel interventions for smoking cessation. Cochrane Database of Systematic Reviews 2004, 1: CD003698.

8. National Heart Foundation of Australia and the Cardiac Society of Australia and New Zealand: Position Statement on Lipid Management-2005. Heart Lung Circ 2005, 14:275-91.

9. National Vascular Disease Prevention Alliance: Guidelines for the assessment of absolute cardiovascular disease risk. National Heart Foundation of Australia 2009 [http://www.heartfoundation.org.au/ Professional_Information/General_Practice/Pages/AbsoluteRisk.aspx], Downloaded 28 April 2010.

10. National Heart Foundation of Australia (National Blood Pressure and Vascular Disease Advisory Committee): Guide to management of hypertension. 2008.

11. New Zealand Guideline Group: The Assessment and Management of Cardiovascular Risk. 2003 [http://www.nhf.org.nz/files/CVD_Risk_Full.pdf], Downloaded 28 April 2010

12. Graham I, Atar D, Borch-Johnsen K, Boysen G, Burell G, Cifkova R, Dallongeville J, De Backer G, Ebrahim S, Gjelsvik B, Herrmann-Lingen C, Hoes A, Humphries S, Knapton M, Perk J, Priori SG, Pyorala K, Reiner Z, Ruilope L, Sans-Menendez S, Scholte op Reimer W, Weissberg P, Wood D, Yarnell J, Zamorano JL, Walma E, Fitzgerald T, Cooney MT, Dudina A: European guidelines on cardiovascular disease prevention in clinical practice: executive summary. Eur Heart J 2007, 28(19):2375-414.

13. World Health Organisation: Prevention of cardiovascular disease: guidelines for assessment and management of total cardiovascular risk. Geneva 2007.

14. Clifford RM, Davis WA, Batty KT, Davis TME: Effect of a Pharmaceutical Care Program on Vascular Risk Factors in Type 2 Diabetes. Diabetes Care 2005, 28(4):771-76.

15. Krass I, Armour CL, Mitchell B, Brillant M, Dienaar R, Hughes J, Lau P, Peterson G, Stewart K, Taylor S, Wilkinson J: The Pharmacy Diabetes Care
Program: assessment of a community pharmacy diabetes service model in Australia. Diabet Med 2007, 24(6):677-83.

16. Emerson L, Krass I: A Collaborative, Interdisciplinary Evidence Based Approach to Reducing Coronary Heart Disease in Rural Areas. Canberra: Pharmacy Guild of Australia 2006.

17. Anderson KM, Odell PM, Wilson PW, Kannel WB: Cardiovascular disease risk profiles. Am Heart J 1991, 121(1 Pt 2):293-8.

18. Cholestech Corporation: Clinical performance of the Cardiocheck P.A and the Cholestech LDX System compared to a Clinical Diagnostic Laboratory Reference Method for the Determination of Lipid Profiles. 2002 [http://www.cholestech.com/docs/ldx_accuracy/tbCardioChek.pdf], Downloaded 28 April 2010

19. Santee J: Accuracy and precision of the Cholestech LDX system in monitoring blood lipid levels. Am J Health Syst Pharm 2002, 59:1774-1779.

20. Tolonen H, Kuulasmaa K, Laatikainen T, Wolf H: European Health Risk Monitoring Project. Recommendation for indicators, international collaboration, protocol and manual of operations for chronic disease risk factor surveys. 2002.

21. WHO MONICA Project Principal Investigators: The World Health Organization MONICA Project (monitoring trends and determinants in cardiovascular disease): a major international collaboration. J Clin Epidemiol 1988, 41(2):105-14.

22. Laatikainen $T$, Janus $E$, Kilkkinen A, Heistaro S, Tideman $P$, Baird A, Tirimacco R, Whiting M, Franklin L, Chapman A, Kao-Philpot A, Dunbar JA: Chronic Disease Risk Factors in Rural Australia: Results From the Greater Green Triangle Risk Factor Surveys. Asia Pac J Public Health 2009, 21(1):51-62.

23. George J, Mackinnon A, Kong DCM, Stewart K: Development and validation of the Beliefs and Behaviour Questionnaire (BBQ). Patient Education and Counseling 2006, 64(1-3):50-60.

24. Morisky DE, Green LW, Levine DM: Concurrent and predictive validity of a self-reported measure of medication adherence. Med Care 1986, 24(1):67-74.

25. Kinect Australia for the Lifescripts consortium: Lifescripts Practice Manual: Supporting Lifestyle Risk Factor Management in General Practice. Canberra: Commonwealth of Australia 2005.

26. National Health and Medical Research Council: Dietary Guidelines for Australian Adults. Canberra 2003.

27. McLennan W, Podger A: National Nutrition Survey: Nutrient Intakes and Physical Measurements Australia 1995. Canberra: Australian Bureau of Statistics 1998.

28. Hodge A, Patterson AJ, Brown WJ, Ireland P, Giles G: The Anti Cancer Council of Victoria FFQ: relative validity of nutrient intakes compared with weighed food records in young to middle-aged women in a study of iron supplementation. Aust N Z J Public Health 2000, 24(6):576-83.

29. Bush K, Kivlahan DR, McDonell MB, Finn SD, Bradley KA, for the Ambulatory Care Quality Improvement Project: The AUDIT Alcohol Consumption Questions (AUDIT-C): An Effective Brief Screening Test for Problem Drinking. Arch Intern Med 1998, 158(16):1789-95.

30. Radloff LS: The CES-D scale: A self-report depression scale for research in the general population. Appl Psychol Meas 1977, 385-401.

31. Ware JE, Kosinski M, Turner-Bowker DM, Gandeck B: User's manual for the SF-12v2 ${ }^{\mathrm{TM}}$ Health Survey. Boston: Quality Metrics 2007

32. Schwarzer R: Modeling Health Behavior Change: How to Predict and Modify the Adoption and Maintenance of Health Behaviors. Appl Psychol 2008, 57(1):1-29.

33. McNaughton SA, Dunstan DW, Ball K, Shaw J, Crawford D: Dietary Quality Is Associated with Diabetes and Cardio-Metabolic Risk Factors. J Nutr 2009, 139(4):734-42.

34. Patel KC, Minhas R, Gill P, Khunti K: Vascular Risk Checks in the UK: strategic challenges for implementation. Heart 2009, (online), hrt.2008.165035.

35. Horgan JMP, Blenkinsopp A, McManus RJ: Evaluation of a cardiovascular disease opportunistic risk assessment pilot ('Heart MOT' service) in community pharmacies. J Public Health 2009, (online), fdp092.

36. Hourihan F, Krass I, Chen T, Hourihan F, Krass I, Chen T: Rural community pharmacy: a feasible site for a health promotion and screening service for cardiovascular risk factors. Aust J Rural Health 2003, 11(1):28-35.

37. Krass I, Hourihan F, T C: Health promotion and screening for cardiovascular risk factors in NSW: a community pharmacy model. Health Promot J Austr 2003, 14(2):101-07. 
38. Absetz P, Valve R, Oldenburg B, Heinonen $H$, Nissinen A, Fogelholm M, Ilvesmaki V, Talja M, Uutela A: Type 2 Diabetes Prevention in the 'Real World'. Diabetes Care 2007, 30(10):2465-70.

39. Laatikainen T, Dunbar JA, Chapman A, Kilkkinen A, Vartiainen E, Heistaro S,

et al: Prevention of type 2 diabetes by lifestyle intervention in an

Australian primary health care setting: Greater Green Triangle (GGT)

Diabetes Prevention Project. BMC Public Health 2007, 7:249.

\section{Pre-publication history}

The pre-publication history for this paper can be accessed here: http://www.biomedcentral.com/1472-6963/10/264/prepub

doi:10.1186/1472-6963-10-264

Cite this article as: Mc Namara et al.: Engaging community pharmacists

in the primary prevention of cardiovascular disease: protocol for the

Pharmacist Assessment of Adherence, Risk and Treatment in

Cardiovascular Disease (PAART CVD) pilot study. BMC Health Services

Research 2010 10:264.

\section{Submit your next manuscript to BioMed Central} and take full advantage of:

- Convenient online submission

- Thorough peer review

- No space constraints or color figure charges

- Immediate publication on acceptance

- Inclusion in PubMed, CAS, Scopus and Google Scholar

- Research which is freely available for redistribution

Submit your manuscript at www.biomedcentral.com/submit 